\title{
Design and Fabrication of Direct Methanol Fuel Cell Current Collector with Temperature Sensors
}

\author{
Yean-Der Kuan, ${ }^{1 *}$ Chung-Tang Liu, ${ }^{1}$ and Min-Feng Sung ${ }^{2}$ \\ ${ }^{1}$ National Chin-Yi University of Technology, \\ No. 35, Lane 215, Section 1, Chung-Shan Road, Taiping Dist., 411 Taichung, Taiwan \\ ${ }^{2}$ Kenda Rubber Ind. Co., Ltd. Sec. 1, Chung Shan Rd., Yuan-Lin, Taiwan, Taiwan
}

(Received January 18, 2018; accepted August 22, 2018)

Keywords: direct methanol fuel cell, micro-electromechanical, resistance temperature detector, current collector

Temperature is an important parameter that affects the direct methanol fuel cell (DMFC) performance, so a system that controls and monitors the DMFC inner temperature would be helpful. The temperature sensors generally added into DMFCs are seldom embedded into bipolar plates, which might increase the device assembly complexity. In this study, we adopt the micro-electromechanical system (MEMS) technique to construct resistance temperature detector (RTD) sensors onto current collectors to increase the measurement accuracy and reduce the DMFC assembly complexity. The applicable measurement range of the RTD sensors fabricated onto the current collectors is designed to be between 30 and $60{ }^{\circ} \mathrm{C}$. The sensitivity of the RDT sensors is about $0.5 \Omega /{ }^{\circ} \mathrm{C}$. The developed sensor adopts a plug-in design to enhance measurement convenience.

\section{Introduction}

Methanol is characterized by high energy density, low price, easy acquisition, and portability. Direct methanol fuel cells (DMFCs) using methanol as fuel are free from the need for an external reformer and hydrogen purification unit. ${ }^{(1)}$ This allows the system to be easily miniaturized. When a DMFC is applied to portable device electronic equipment as a power source, it must be light, thin, and small. The research and development of DMFCs is therefore coupled with microminiaturization and lightweight requirements. Temperature is an important parameter that affects DMFC performance. Fuel cell internal temperature monitoring contributes to DMFC system control. In this study, we use the micro-electromechanical system (MEMS) technique to construct a resistance temperature detector (RTD) onto a lightweight current collector plate to integrate the temperature monitoring mechanism with DMFC bipolar plates while maintaining the light weight and microminiaturization requirements.

*Corresponding author: e-mail: ydkuan@ncut.edu.tw https://doi.org/10.18494/SAM.2018.2021 


\section{Temperature Sensor Types}

There are numerous types of electron temperature probes, with the most common types being bimetallic thermometers based on the thermal expansion principle and monometallic RTDs or nonmetallic thermistors. The former is a type of sensor based on the principle of different thermal expansion coefficients from two metals. Two metals have different degrees of expansion at the same temperature, such that as a metal strip bends, the curvature is measured to estimate the temperature. The curvature is proportional to the length square and temperature difference of the metal strip, and inversely proportional to the thickness. As the metal lath expansion curvature is very small, it is often made into a helical tube in order to increase the curvature. The latter medium can be divided into metal and nonmetal types. The monometal impedance varies with the temperature, so it can be used to estimate the temperature. Platinum, copper, or nickel are the most commonly used metals, coiled at an appropriate wire thickness to form a resistor, or made into a film for measurement. The common RTD has the following advantages: (1) very high linearity, (2) wide operating range, (3) hot operation, and (4) superhigh stability even at high temperatures, and the platinum film type has a shorter response time. Another type for measuring temperature is the thermistor that is nonmetal and semiconductive. It is characterized by negative and positive temperature coefficients according to the temperature relation. The thermistor refers mostly to a negative temperature coefficient (NTC). Its main defect is nonlinearity, so it needs a circuit to convert the response linear terms, which may reduce its sensitivity. Another common type is the thermocouple based on the thermal electromotive force principle. Two alloy wires at different uniformities are combined and two nodes are used as hot point and instrument node cold point references (reference point). When a closed loop is formed with the instrument and the temperatures at the two points are different, a current is produced inside. If an open circuit is formed, the voltage can be measured to estimate the temperature. ${ }^{(2)}$ Therefore, there are many temperature measurement methods. In this research, we construct a temperature sensor onto a collector plate using an electromechanical process.

\section{Lightweight Current Collector Plate with Temperature Sensor}

\subsection{Lightweight current collector plate}

A fuel cell produces low voltage and high current, requiring a stack of fuel cells to obtain the appropriate operating voltage. Barranco et al. used the cathodic arc evaporation physical vapor deposition (CAE-PVD) micro-electromechanical process to deposit $\mathrm{Zr}$ and $\mathrm{Cr}$ metals onto aluminum (AL5083)-based collector plates. ${ }^{(3)} \mathrm{As} \mathrm{Zr}$ is unlikely to corrode, it is used in nuclear reactors to absorb neutrons and as a corrosion-resistant alloy. $\mathrm{Cr}$ is also a common corrosionresistant metal material. In their study, they discussed the effect of the Al collector plate with the two corrosion-resistant metals on the hydrogen-oxygen fuel cell performance. The results showed that the collector plate accounted for at least $80 \%$ of the overall fuel cell weight and $40 \%$ of the overall pile cost. Therefore, developing a lightweight, low-cost collector plate 
has become a very important subject of fuel cell research. Yun reported that $\mathrm{Au}-\mathrm{Ti}$ and $\mathrm{Au}-$ Ni metal films deposited onto the surface of stainless steel collector plates could reduce the collector plate impedance, improving the cell performance. ${ }^{(4)}$ Yi et al. found that the stainless steel SS304 with a carbon coating could be used as a fuel cell collector plate. ${ }^{(5)}$ The SS304 used in the literature was $100 \times 100 \mathrm{~mm}^{2}$ in size and only $0.1 \mathrm{~mm}$ in thickness. The Closed Field Unbalanced Magnetron Sputter Ion Plating (CFUBMSIP) deposited 99.99\% pure graphite and $\mathrm{Cr}$ onto the stainless steel surface by sputtering in a chamber filled with $99.99 \%$ argon and nitrogen. The impedance test showed that the contact resistance of the SS304 stainless steel collector plate with a carbon coating was $5.4 \mathrm{~m} \Omega \mathrm{cm}^{-2}$, and that without a carbon coating was $93.3 \mathrm{~m} \Omega \mathrm{cm}^{-2}$. Lee et al. used the MEMS PVD and deep reactive ion etching (DRIE) processes to make a micro-DMFC. They used the DRIE process to make 10-, 30-, and 50- $\mu$ m-deep and 836- $\mu \mathrm{m}$-wide runners, and the PVD process to deposit $70 \mathrm{~nm} \mathrm{Pt}$ and $20 \mathrm{~nm}$ Ti onto the runner surface as the collector and temperature microsensor, respectively. ${ }^{(6)}$ The temperature microsensor was embedded in the DMFC. The measurement result showed that the resistivity was $7.524-7.677 \mathrm{k} \Omega$, and the measurable temperature range was $20-46{ }^{\circ} \mathrm{C}$.

In summary, better bipolar or collector plates must have high electrical conductivity. Therefore, the earliest fuel cell bipolar plates were made of graphite. However, graphite is fragile and expensive, and it is averse to microminiaturization and lightweight fuel cells. Therefore, the stainless steel SS316, SS304, Al, or integrated The printed circuit board (PCB) processes were utilized in fuel cell bipolar or collector plates. In recent years, the MEMS technique has matured, and bipolar or collector plate electrical conductivity can be enhanced using the MEMS process to deposit various metallic films onto the stainless steel surface. The Ti-based collector plate has become a topical subject. PCB process application can reduce the fuel cell weight considerably. However, nonmetal-based bipolar or collector plates are still rare. The main advantages of the nonmetal base are low cost and light weight. ${ }^{(7)}$ Therefore, in this study, we developed a lightweight current collector plate on the basis of a previous study and attached a temperature sensor to the back side of the lightweight current collector plate to measure the exact fuel cell temperature in the experimental process. ${ }^{(8)}$

\subsection{Design and fabrication of current collector with RTDs}

The RTD embedded in the lightweight current collector is made by the MEMS process. There are six processes involved in current collector production, namely, cleaning, photoresist coating, exposure, development, sputtering, and metal lift off, as shown in Fig. 1. The substrate surface is cleaned with acetone, which is rinsed off rapidly using pure water to avoid acetone residue. The majority of the water is then blown off using nitrogen. The substrate is then placed on a $90{ }^{\circ} \mathrm{C}$ baking tray for $10 \mathrm{~min}$ to remove the moisture completely. An automatic spin coater is used to coat the substrate with the photoresist AZ4620 by spinning. The photoresist is dried automatically in an automatic spin coater. The coated substrate is then placed onto a custom-made 6" acrylic stage. This acrylic stage has two grooved layers. The lower layer is the size of a 6" wafer. Its height is $0.4 \mathrm{~mm}$, which matches the substrate thickness. There is a plain edge gripping groove for placing the substrate and a mechanism to eject the substrate upward 


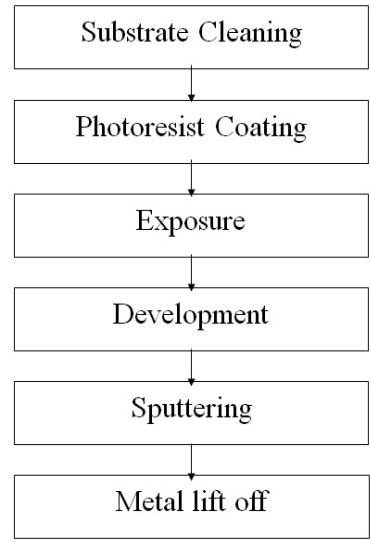

Fig. 1. RTD fabrication process.

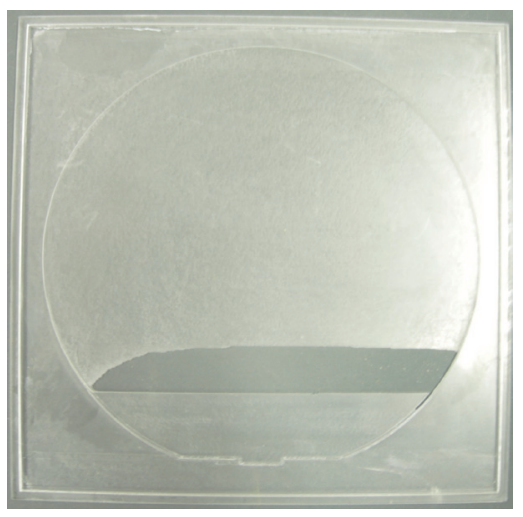

Fig. 2. (Color online) Acrylic stage.

to take the mask. The upper layer is a square grooved area for mask placement $\left(163.9 \mathrm{~mm}^{2}\right)$, as shown in Fig. 2. It is covered with the self-designed mask. The stage, substrate, and mask are put into an automatic aligner and exposed for $5 \mathrm{~s}$ through manual alignment. The exposed substrate is placed into glassware filled with the AD-10 developer. The glassware is shaken manually to remove the remaining photoresist. The developer and residual photoresist are then washed away with a large amount of water. The device is then blow-dried using a nitrogen gun. The developed substrate is then fed into a sputtering machine. The Al-1kW-2 $\mathrm{k} \AA$ process is selected for the machine. The sputtered substrate is then placed into glassware filled with acetone. The remaining photoresist is shaken off using an ultrasonic cleaning machine. The RTD is now completed.

The RTD is usually made on a very smooth surface glass or silicon chip. In this study, however, we produced the RTD on a composite material with a relatively rough surface. In order to obtain the appropriate wire thickness, SU8 was used as the photoresist in the initial stage. The composite material adhesion was poor in multiple experiments and the process development was very likely to fail. The RTD made with the AZ4620 photoresist in the intermediate stage was likely to be affected by subtle changes in contact resistance, which would distort the measurement values. Therefore, in order to improve this phenomenon, the sensing length and aspect ratio were increased. The final design, which was developed after multiple trials and tests, is shown in Fig. 3. The RTD design uses a wire of $200 \mu \mathrm{m}$ thickness and $89 \mathrm{~mm}$ length with a 445 aspect ratio. In addition, in order to monitor the temperatures at the fuel runner inlet/outlet and runner midsection, four sets of RTDs are set to monitor the corresponding temperatures. The completed mask design is shown in Fig. 4. The completed lightweight current collector plate with the temperature sensor is shown in Fig. 5. Figure 5(a) shows the electrode side of the lightweight current collector plate, and Fig. 5(b) shows the other side embedded with the RTD. 


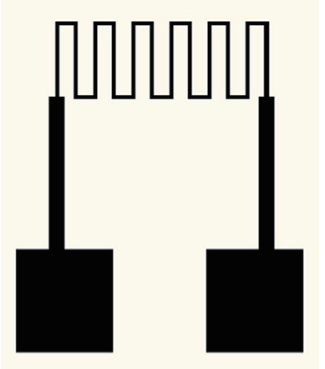

Fig. 3. (Color online) RTD sensor design.

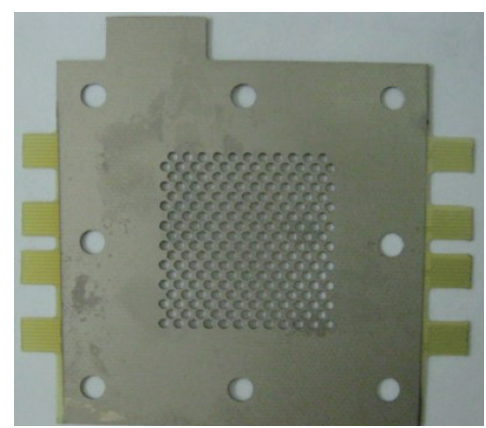

(a)

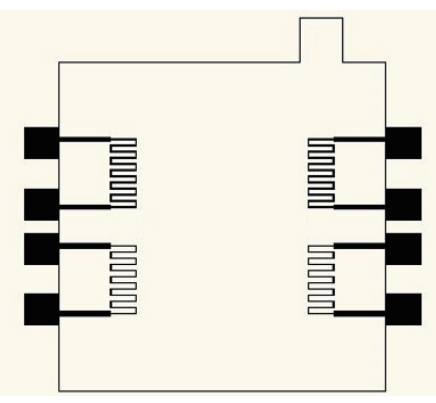

Fig. 4. (Color online) RTD sensor mask design.

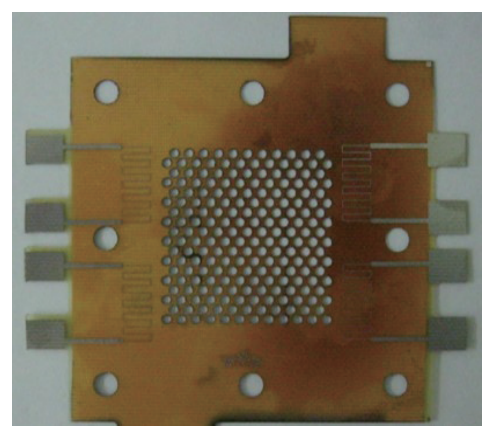

(b)

Fig. 5. (Color online) (a) Electrode and (b) RTD sides of the lightweight current collector.

\section{Experimental Procedure}

\subsection{Linear relationship between temperature and resistance of the RTD}

In order to validate the resistance values corresponding to the temperature microsensor designed and made in this study at different temperatures, a testing architecture was planned as shown in Fig. 6. Figure 6 shows a schematic diagram of the experiment construction for testing the linear relationship between the RTD temperature and impedance. The lightweight current collector plate with the temperature sensor was put into a heat press machine with the sensing end downward and fixed with a water-cooled chuck. A water chiller conveyed cold/hot water to the lower water-cooled chuck (the water chiller had a heating function), the heat press machine provided assistance (for a constant high temperature), and the temperature wire of a commercially available electronic thermometer was embedded between the lower water-cooled chuck and the current collector plate to monitor the temperature. A picture of the experimental setup is shown in Fig. 7. An NI-PXI1033 multifunctional electric meter from National Instruments and the data acquisition program developed by our research team were used for recording. The heat press machine temperature was regulated to $35,40,45,50,55$, and $60{ }^{\circ} \mathrm{C}$, and the water chiller was adjusted when the temperature was too high or too low as indicated by 


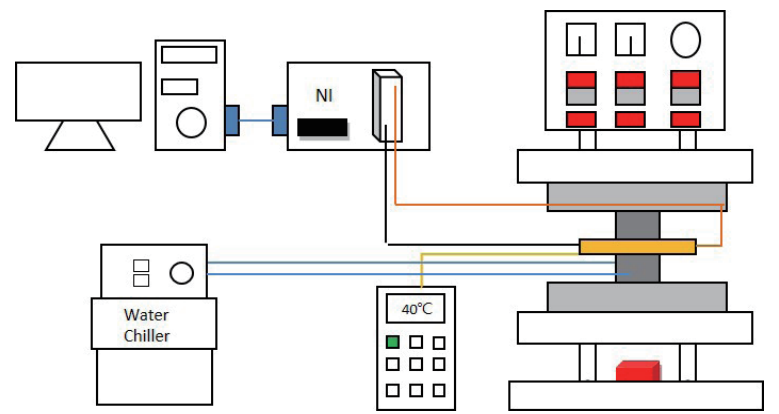

Fig. 6. (Color online) Schematic diagram of the experimental setup for determining the linear relationship between temperature and resistance of RTD sensor.

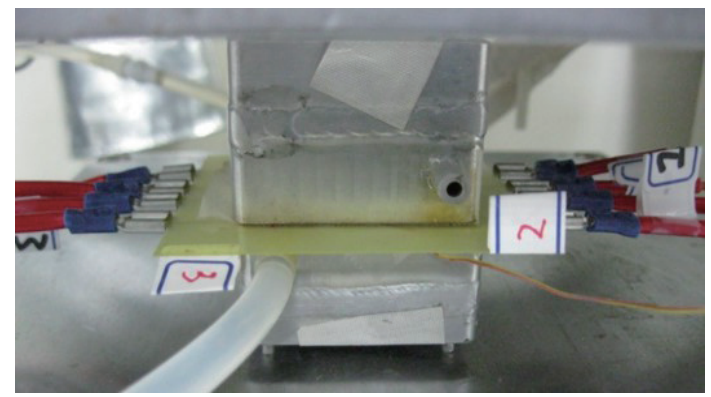

Fig. 7. (Color online) Picture of experimental setup for determining the linear relationship between temperature and resistance of RTD sensor.

the thermometer. One data was recorded per second and the impedance of various RTDs was recorded. The recording lasted $600 \mathrm{~s}$ at different temperatures and the average was taken. The average impedances at various temperatures were drawn into the linear relationship between the RTD temperature and resistance.

Figure 8 shows the experimental results of this RTD sensor when the temperature was set at $35,40,45,50,55$, and $60{ }^{\circ} \mathrm{C}$. The resistance values for various sensors and the linear relationship concluded from the experimental results are as follows.

$$
\begin{aligned}
& \text { Ch1: } y=(x-198.73) / 0.2993 \\
& \text { Ch2: } y=(x-195.7) / 0.3796 \\
& \text { Ch3: } y=(x-223.63) / 0.4803 \\
& \text { Ch4: } y=(x-217.8) / 0.4781
\end{aligned}
$$

where $y$ is the temperature $\left({ }^{\circ} \mathrm{C}\right)$ and $x$ is the resistance $(\Omega)$.

\subsection{Performance measurement of DMFC with lightweight current collector with RTD sensors}

Figure 9 shows a schematic diagram of the experimental setup for the performance measurement of the DMFC with a lightweight current collector with RTD sensors. The DMFC with the collector plate with a temperature sensor and a runner chuck, and a constanttemperature water tank were put in a constant-temperature and constant-humidity machine. A beaker with a water/methanol mixture was heated in the constant-temperature water tank. The constant-humidity and constant-temperature machine was set at 35,45 , and $55{ }^{\circ} \mathrm{C}$ at a constant relative humidity of $60 \%$. Heated liquid methanol was fed using a peristaltic pump in the DMFC anode and air was fed using an air pump in the cathode. The DMFC polarization curves and temperature were then measured using RTD sensors. 


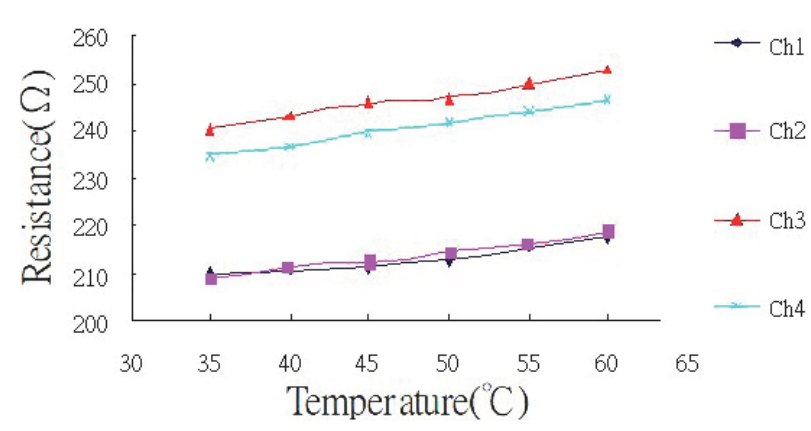

Fig. 8. (Color online) Linear relationship between RTD temperature and resistance.

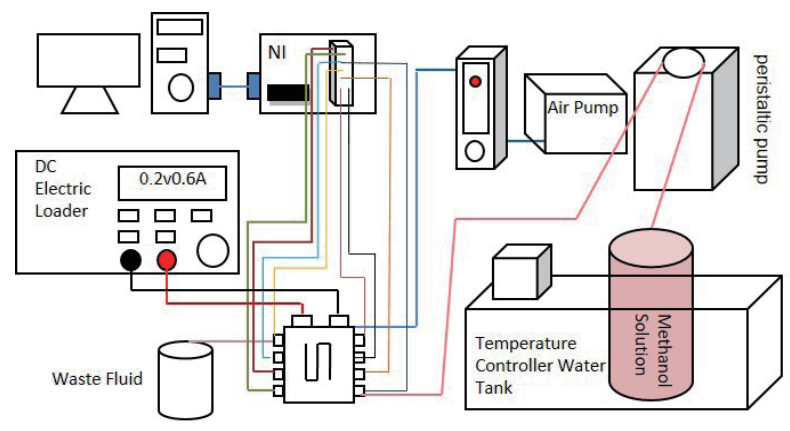

Fig. 9. (Color online) Schematic diagram of experimental setup for the performance measurements of DMFC.

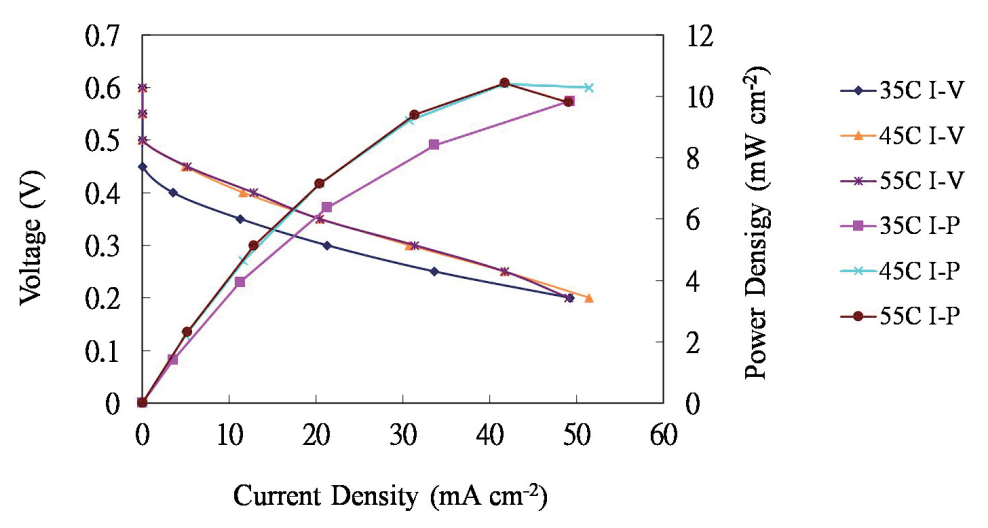

Fig. 10. (Color online) DMFC cell performance at different temperatures.

When the RTD was calibrated, the lightweight current collector plate with RTD temperature sensors was assembled in the DMFC for performance testing. From the experimental results, the cell performance characteristics at different temperatures are shown in Fig. 10. When the methanol temperatures were 35,45 , and $55{ }^{\circ} \mathrm{C}$, the current densities of the cell were 21.22, 30.69 , and $31.34 \mathrm{~mA} \mathrm{~cm}^{-2}$, respectively, when the load voltage was $0.3 \mathrm{~V}$. Table 1 shows the measured resistance values for the lightweight collector plate with RTD temperature sensors in the experiment. The higher the methanol input temperature, the higher the resistance measured by the RTD in the fuel cell collector plate. The experimental data in this table were substituted in Eqs. (1)-(4) to obtain the experimental results shown in Table 2. It was observed that, even when the fuel cell was put in the constant-humidity and constant-temperature machine, and the methanol solution was heated by excluding water to control the temperature, there was a slight difference observed in the actual fuel cell reaction. The temperature measured by RTD No. Ch2 straight above the input end of the fuel cell was even $65^{\circ} \mathrm{C}$, such that the DMFC might be damaged. Therefore, if the RTD sensors can be located in the area where the thermometer probe cannot be placed, the actual DMFC operating temperature can be measured, such that the fuel cell operating temperature can be mastered in future DMFC stack operations and the DMFC operational stability and performance can be improved using peripheral control strategies. 
Table 1

Resistance values measured using lightweight collector plate with RTD sensors under different methanol solution temperatures.

\begin{tabular}{lcccc}
\hline Methanol solution & \multicolumn{4}{c}{ RTD sensor $(\Omega)$} \\
\cline { 2 - 5 } temperature $\left({ }^{\circ} \mathrm{C}\right)$ & Ch1 & Ch2 & Ch3 & Ch4 \\
\hline 35 & 208.9 & 210.1 & 242.0 & 234.7 \\
45 & 210.6 & 214.3 & 245.3 & 238.9 \\
55 & 218.3 & 221.5 & 250.2 & 243.7 \\
\hline
\end{tabular}

Table 2

Temperature values measured using lightweight collector plate with RTD sensors under different methanol solution temperatures.

\begin{tabular}{lllll}
\hline Methanol solution & \multicolumn{4}{c}{ RTD sensor $\left({ }^{\circ} \mathrm{C}\right)$} \\
\cline { 2 - 5 } temperature $\left({ }^{\circ} \mathrm{C}\right)$ & Ch1 & Ch2 & Ch3 & Ch4 \\
\hline 35 & 34.0 & 37.8 & 38.2 & 35.3 \\
45 & 39.7 & 49.0 & 45.2 & 44.2 \\
55 & 65.5 & 68.0 & 55.3 & 54.1 \\
\hline
\end{tabular}

\section{Conclusions}

A strategy for embedding RTD sensors, fabricated using the MEMS technique, into DMFC current collectors is proposed. A prototype of the lightweight current collector with four RTDs was successfully fabricated and assembled into a DMFC. The experimental results demonstrated good linear relationship between the temperature and resistance of the RTD sensors. Further temperature measurements on the DMFC operating under different methanol solutions were also conducted and the feasibility of the proposed strategy was verified.

\section{Acknowledgments}

The authors would like to acknowledge the financial support from the Ministry of Science and Technology of Taiwan throughout the research.

\section{References}

1 R. O’Hayre, S.-W. Cha, W. Colella, and F. B. Prinz: Fuel Cell Fundamentals (Wiley, New Jersey, 2005) 2nd ed.

2 L. Wu: Temperature Sensor-Theory and Application (Chuan Hwa Books, Taiwan, 1990).

3 J. Barranco, F. Barreras, A. Lozano, A. M. Lopez, V. Roda, J. Martin, M. Maza, G. G. Fuentes, and E. Almandoz: Int. J. Hydrogen Energy 35 (2010) 11489. https://doi.org/10.1016/j.ijhydene.2010.05.050

4 Y. H. Yun: Int. J. Hydrogen Energy 35 (2010) 1713. https://doi.org/10.1016/j.ijhydene.2009.12.036

5 P. Yi, L. Peng, L. Feng, P. Gan, and X. Lai: J. Power Sources 195 (2010) 7061. https://doi.org/10.1016/ j.jpowsour.2010.05.019

6 C. Y. Lee, R. D. Huang, and C. W. Chuang: Jpn. J. Appl. Phys. 46 (2007) 6911. https://doi.org/10.1143/ JJAP.46.6911

7 C. Ayranci and J. Carey: Compos. Struct. 85 (2008) 43. https://doi.org/10.1016/j.compstruct.2007.10.004

8 M.-F. Sung, Y.-D. Kuan, B.-X.Chen, and S.-M. Lee: J. Power Sources 196 (2011) 5897. https://doi.org/10.1016/ j.jpowsour.2011.02.082 